\title{
Association between hypoxemia and anemia following arthroplasty: A pilot clinical study
}

\author{
FUQIANG GAO, WEI SUN, WANSHOU GUO, LIMING CHENG, ZIRONG LI and NEPALI KUSH \\ Osteonecrosis and Joint-Preserving and Reconstruction Center, Department of Orthopaedic Surgery, \\ China-Japan Friendship Hospital, Beijing 100029, P.R. China
}

Received December 25, 2014; Accepted February 11, 2016

DOI: 10.3892/etm.2016.3113

\begin{abstract}
Hypoxia and anemia are common complications following joint arthroplasty. Whether hypoxia indicates that a patient is anemic and whether anemia causes a decline in arterial oxygen pressure accompanied by hypoxemia are not completely understood. The aim of the present study was to determine the association between hypoxemia and anemia following arthroplasty. A total of 135 patients who underwent arthroplasty at the China-Japan Friendship Hospital between January and May 2013 were retrospectively analyzed. The patients were divided into five groups depending on the type of arthroplasty they had experienced: Unilateral total knee arthroplasty (TKA), bilateral TKA, unilateral total hip arthroplasty (THA), bilateral THA or unilateral unicompartmental knee arthroplasty. Perioperative peripheral oxygen saturation $\left(\mathrm{SpO}_{2}\right)$ and hemoglobin $(\mathrm{Hb})$ levels were assessed, and the associations between the changes in $\mathrm{SpO}_{2}\left(\Delta \mathrm{SpO}_{2}\right)$ and hemoglobin $(\Delta \mathrm{Hb})$ levels on the first and third postoperative days (PODs) were analyzed using Pearson's correlation test for each group. The perioperative $\mathrm{SpO}_{2}$ curves for the various groups were typically at their lowest on the day of surgery. Significant hypoxemia was observed on POD 0-2, although a stable recovery curve was observed on POD 3-5. Trends in $\triangle \mathrm{Hb}$ were observed among the 5 groups, with the lowest $\mathrm{Hb}$ value observed predominantly on POD 2 and 3. By POD 4 and $5 \mathrm{Hb}$ levels had recovered, with a steadily and consistently increasing curve. There was no statistically significant correlation between $\Delta \mathrm{SpO}_{2}$ and decrease in $\mathrm{Hb}$ levels $(\mathrm{P}>0.05) . \mathrm{SpO}_{2}$ levels should not serve as a clinical indicator of the incidence and severity of anemia in patients who have undergone primary arthroplasty. To a point, the degree of postoperative anemic status does not affect $\mathrm{SpO}_{2}$ levels.
\end{abstract}

Correspondence to: Dr Wei Sun, Osteonecrosis and Joint-Preserving and Reconstruction Center, Department of Orthopaedic Surgery, China-Japan Friendship Hospital, 2 Yinghua Dongjie, Hepingli, Beijing 100029, P.R. China

E-mail: 18901267995@163.com

Key words: arthroplasty, replacement, knee, hip, postoperative complications, hypoxemia, anemia

\section{Introduction}

Hypoxemia following arthroplasty has been reported to be a common postoperative complication (1-3). In $32 \%$ of hypoxic patients, hypoxemia was the initial symptom to develop and is a predicting factor for major complications, and is defined as life-threatening if left untreated (1-3). It has been suggested that a hypoxemic episode may contribute to myocardial ischemia, infarction, wound infection $(2,4,5)$, mental confusion $(6,7)$, heterotopic ossification and idiopathic arthrofibrosis (8) following the surgical procedure. Myocardial ischemia is more likely to occur if an episode of hypoxemia is prolonged $(>5 \mathrm{~min})$ and severe [perioperative peripheral oxygen saturation $\left.\left(\mathrm{SpO}_{2}\right)<85 \%\right]$ (5). The morbidity associated with hypoxia is exacerbated by delirium, which commonly follows total hip arthroplasty (THA) in patients with femoral neck fractures (7).

Anemia is commonly encountered during the perioperative period $(3,5,7)$. Blood loss may occur from the osteotomy surfaces of the distal femoral and proximal tibial bones, from the soft tissue-injury and from the dredged marrow cavity (9). However, long-term anticoagulation therapy and early rehabilitation of joint function have also resulted in postoperative anemia (10).

Questions persist as to whether hypoxia indicates that a patient is anemic and whether anemia causes a decline in arterial oxygen pressure accompanied by hypoxemia. The purpose of the present study was to determine the association between hypoxemia and anemia following arthroplasty.

\section{Materials and methods}

Ethical approval. The present study was approved by the Institutional Review Board on Human Studies of the Ethical Committee of the China-Japan Friendship Hospital (Beijing. China), and was conducted in accordance with the Helsinki Declaration of 1975 (11). All patients were required to sign an informed consent form prior to participation in the study. The written-informed consent forms were provided by the patients and stored in the hospital database for clinical research.

Patient characteristics. A total of 135 patients (male, 53; female, 82; mean age, 59.7 years; age range, 24-83 years) who underwent arthroplasty at the China-Japan Friendship Hospital from January to May 2013 were retrospectively studied. The 
Table I. Characteristics of the patients who underwent arthroplasty.

\begin{tabular}{|c|c|c|c|c|c|}
\hline Characteristic & U-THA & B-THA & U-TKA & B-TKA & U-UKA \\
\hline Number of patients & 51 & 15 & 42 & 22 & 5 \\
\hline Gender (male/female) & $33 / 18$ & $8 / 7$ & $7 / 35$ & $5 / 17$ & $0 / 5$ \\
\hline Age (years) & $54.3 \pm 13.5$ & $47.3 \pm 13.3$ & $67.1 \pm 8.4$ & $66.0 \pm 7.4$ & $59.8 \pm 9.5$ \\
\hline Height (cm) & $165.9 \pm 6.9$ & $165.3 \pm 5.7$ & $161.5 \pm 6.5$ & $163.2 \pm 7.2$ & $158.8 \pm 4.0$ \\
\hline Weight (kg) & $67.5 \pm 11.5$ & $71.4 \pm 12.4$ & $70.4 \pm 9.7$ & $74.3 \pm 12.3$ & $66.3 \pm 9.5$ \\
\hline BMI $\left(\mathrm{kg} / \mathrm{m}^{2}\right)$ & $24.5 \pm 3.5$ & $26.1 \pm 3.8$ & $26.9 \pm 2.9$ & $27.9 \pm 4.2$ & $26.3 \pm 4.1$ \\
\hline Diagnosis (OA/RA/ONFH) & $12 / 4 / 35$ & 1/0/14 & $42 / 0 / 0$ & $22 / 0 / 0$ & $5 / 0 / 0$ \\
\hline Anesthesia (ESA/GA) & $12 / 39$ & $1 / 14$ & $9 / 33$ & $0 / 22$ & $1 / 4$ \\
\hline Anticoagulants (LMWH/R) & $45 / 6$ & $15 / 0$ & $36 / 6$ & $20 / 2$ & $4 / 1$ \\
\hline
\end{tabular}

U-THA, unilateral total hip arthroplasty; B-THA, bilateral total hip arthroplasty; U-TKA, unilateral total knee arthroplasty; B-TKA, bilateral total knee arthroplasty; U-UKA, unilateral unicompartmental knee arthroplasty; BMI, body mass index; OA, osteoarthritis; RA, rheumatoid arthritis; ONFH, osteonecrosis of the femoral head; ESA, epidural spinal anesthesia; GA, general anesthesia; LMWH, low molecular weight heparin; $\mathrm{R}$, rivaroxaban.

inclusion criteria were as follows: Primary arthroplasty, no previous history of severe lung disease, normal preoperative pulmonary function, and availability of complete medical records. Patients were divided into 5 groups according to the type of arthroplasty they underwent (Table I): Unilateral total knee arthroplasty (TKA), bilateral TKA, unilateral THA, bilateral THA or unilateral unicompartmental knee arthroplasty (UKA). Patient charts were reviewed in order to obtain the required data, including body mass index, preoperative diagnosis, type of anesthesia, medical complications, and anticoagulant options (either low molecular weight heparin or rivaroxaban). Height and weight were recorded preoperatively. $\mathrm{SpO}_{2}$ and hemoglobin $(\mathrm{Hb})$ levels were measured by routine blood tests perioperatively. Room-air $\mathrm{SpO}_{2}$ was measured prior to the surgical procedure using a pulse oximeter (Portable PC-60C Non-invasive Pulse Oximeter; Shenzhen Creative Industry Co., Ltd., Guangdong, China). Oxygen saturation was measured preoperatively, intraoperatively, and on postoperative days (POD) 1-6 using a pulse oximeter. Nursing surveillance included recording of pulse oximetry with routine vital signs every $6 \mathrm{~h}$ following arthroplasty. Hypoxemia was defined as $\mathrm{SpO}_{2}<90 \%$. A complete blood count was performed for all patients pre- and postoperatively via routine blood tests. A detailed data sheet recording the exact circumstances surrounding a hypoxic event, the general information and the final outcome was completed for each patient. No patient developed a pulmonary embolism.

Surgical procedures. All surgical procedures were performed by or under the supervision of a fellowship-trained arthroplasty surgeon. Hip arthroplasty at the China-Japan Friendship Hospital is performed with the patient in the lateral decubitus position using a modified posterolateral approach and uncemented components. TKA and UKA are performed on an exsanguinated limb under tourniquet pressure, using a medial parapatellar arthrotomy and cement fixation (12). The postoperative protocol for arthroplasty patients included deep venous thrombosis prophylaxis with subcutaneous administration of 4,000-6,000 U low molecular-weight heparin (Glaxo
Wellcome Production, Evreux, France) or oral administration of $10 \mathrm{mg}$ rivaroxaban (Bayer AG, Leverkusen, Germany). All arthroplasty patients were placed on oxygen during the immediate postoperative period and weaned over the following $24 \mathrm{~h}$. Patients were mobilized early in the postoperative period and were encouraged to ambulate with assistive devices on POD 2-3.

Statistical analysis. Data are presented as the mean \pm standard deviation. All data analyses were performed using SPSS 16.0 (SPSS, Inc., Chicago, IL, USA). In all patients, $\mathrm{SpO}_{2}$ levels and $\mathrm{Hb}$ were measured and drawn into a tendency chart preoperatively, intraoperatively and on POD 1-6. Variations in these parameters were identified, and associations between the changes in $\mathrm{SpO}_{2}\left(\Delta \mathrm{SpO}_{2}\right)$ and $\mathrm{Hb}(\Delta \mathrm{Hb})$ levels on POD 1 and 3 were analyzed using Pearson's correlation test for each category of arthroplasty. $\mathrm{P}<0.05$ was considered to indicate a statistically significant result.

\section{Results}

The perioperative $\mathrm{SpO}_{2}$ curve for each surgical category demonstrated that $\mathrm{SpO}_{2}$ was typically lowest on the day of surgery (POD 0). Marked hypoxemia was observed on POD 0-2, although the recovery curve demonstrated a stable increase in $\mathrm{SpO}_{2}$ on POD 3-5 (Fig. 1). The analysis of $\Delta \mathrm{Hb}$ revealed trends among the 5 groups, with the lowest $\mathrm{Hb}$ value observed most frequently on POD 2-3 following arthroplasty. $\mathrm{Hb}$ levels increased consistently over POD 4-5 (Fig. 2).

$\Delta \mathrm{SpO}_{2}$ was not associated with $\Delta \mathrm{Hb}(\mathrm{P}>0.05)$. The results of the Pearson's correlation tests between $\Delta \mathrm{SpO}_{2}$ and $\Delta \mathrm{Hb}$ on POD 1 and 3 are shown in Table II. For unilateral THA, the $\Delta \mathrm{SpO}_{2}$ on POD 1 and 3 were $3.9 \pm 1.7$ and $2.7 \pm 1.7 \%$, respectively, and the $\Delta \mathrm{Hb}$ on POD 1 and 3 were 12.6 \pm 9.1 and 27.3 $\pm 9.2 \mathrm{~g} / 1$, respectively. There was a negative correlation between $\Delta \mathrm{SpO}_{2}$ and $\Delta \mathrm{Hb}$ (on POD $1, r=0.236$ and $\mathrm{P}=0.096$; on POD 3, $r=-0.113$ and $\mathrm{P}=0.430)$. Similar results were observed for the bilateral THA (on POD 1, $r=-0.192$ and $\mathrm{P}=0.494$; on POD 3 and $r=-0.168$ and $\mathrm{P}=0.548$ ). For unilateral TKA, the $\Delta \mathrm{SpO}_{2}$ 
Table II. Results of Pearson's correlation tests between $\Delta \mathrm{SpO}_{2}$ and $\Delta \mathrm{Hb}$ on the postoperative first and third days.

\begin{tabular}{lccccc}
\hline Variable & U-THA & B-THA & U-TKA & B-TKA & U-UKA \\
\hline Patient nos. & 51 & 15 & 42 & 22 & 5 \\
Post-op & & & & & \\
$\Delta \mathrm{SpO}_{2}$ & $3.9 \pm 1.7$ & $3.3 \pm 1.5$ & $4.7 \pm 1.8$ & $5.5 \pm 2.3$ & $2.4 \pm 1.5$ \\
$\Delta \mathrm{Hb}$ & $12.6 \pm 9.1$ & $14.0 \pm 14.7$ & $20.0 \pm 10.8$ & $18.8 \pm 9.3$ & $12.6 \pm 9.1$ \\
$r$-value & 0.236 & -0.192 & 0.244 & 0.238 & -0.164 \\
P-value & 0.096 & 0.494 & 0.119 & 0.286 & 0.792 \\
Post-op & & & & & $3.5 \pm 2.2$ \\
$\Delta \mathrm{SpO}_{2}$ & $2.7 \pm 1.7$ & $2.3 \pm 2.3$ & $2.7 \pm 1.7$ & $38.5 \pm 8.4$ & $2.0 \pm 1.6$ \\
$\Delta \mathrm{Hb}$ & $27.3 \pm 9.2$ & $20.5 \pm 11.4$ & $32.3 \pm 11.4$ & 0.374 & 0.942 \\
$r$-value & -0.113 & -0.168 & -0.072 & 0.087 & 0.017 \\
P-value & 0.430 & 0.548 & 0.652 & & \\
\hline
\end{tabular}

U-THA, unilateral total hip arthroplasty; B-THA, bilateral total hip arthroplasty; U-TKA, unilateral total knee arthroplasty; B-TKA, bilateral total knee arthroplasty; U-UKA, unilateral unicompartmental knee arthroplasty; $\mathrm{SpO}_{2}$, perioperative peripheral oxygen saturation; Hb, hemoglobin.

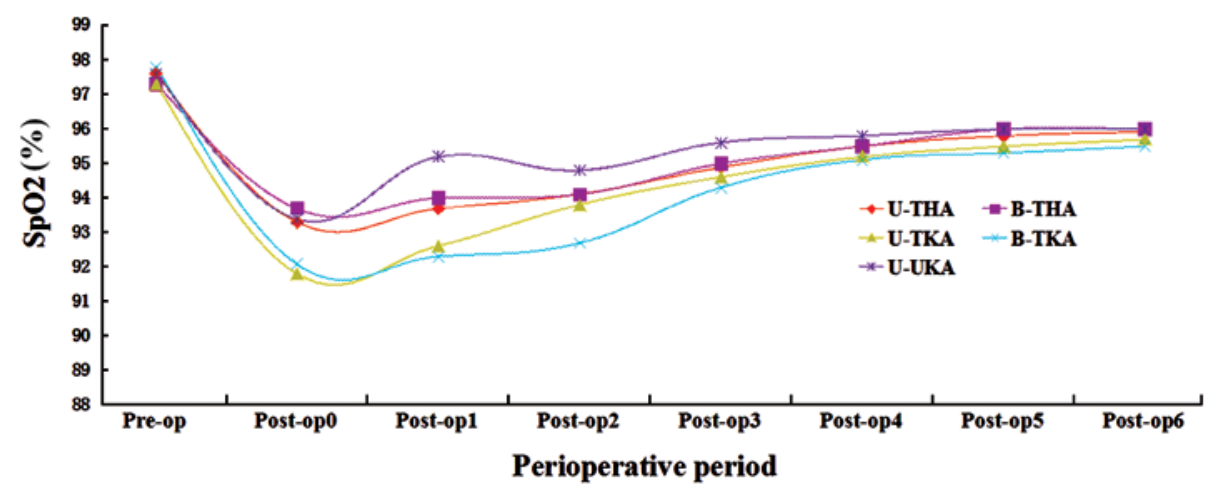

Figure 1. Perioperative $\mathrm{SpO}_{2}$ levels associated with various types of arthroplasty. $\mathrm{SpO}_{2}$, peripheral oxygen saturation; U-THA, unilateral total hip arthroplasty; B-THA, bilateral total hip arthroplasty; U-TKA, unilateral total knee arthroplasty; B-TKA, bilateral total knee arthroplasty; U-UKA, unilateral unicompartmental knee arthroplasty.

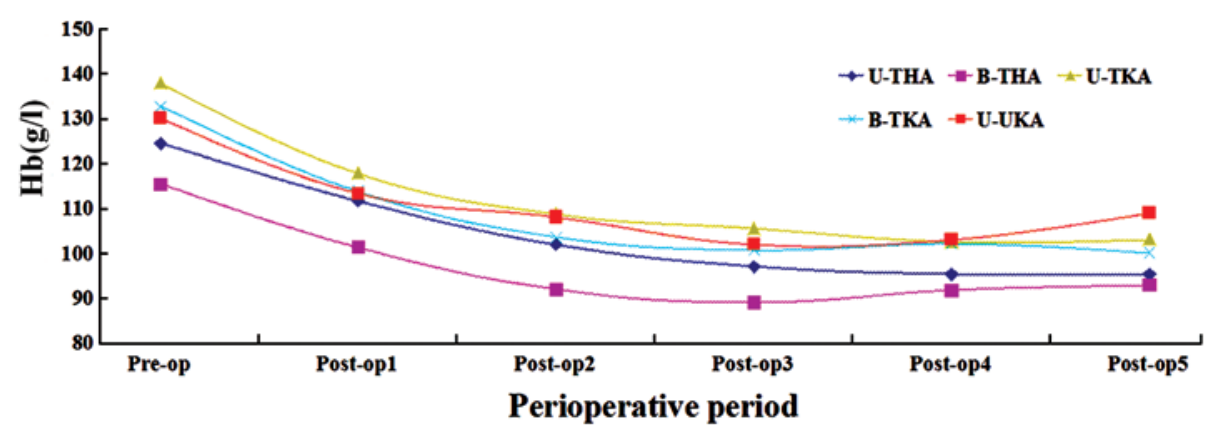

Figure 2. Perioperative hemoglobin levels associated with various types of arthroplasty. Hb, hemoglobulin; U-THA, unilateral total hip arthroplasty; B-THA, bilateral total hip arthroplasty; U-TKA, unilateral total knee arthroplasty; B-TKA, bilateral total knee arthroplasty; U-UKA, unilateral unicompartmental knee arthroplasty.

on POD 1 and 3 were $4.7 \pm 1.8$ and $2.7 \pm 1.7 \%$, respectively, and the $\triangle \mathrm{Hb}$ on POD 1 and 3 were $20.0 \pm 10.8$ and $32.3 \pm 11.4 \mathrm{~g} / \mathrm{l}$, respectively. Correlation analyses showed that the $\Delta \mathrm{SpO}_{2}$ and $\Delta \mathrm{Hb}$ were not closely associated (on POD $1, r=0.244$ and $\mathrm{P}=0.119$; on $\mathrm{POD} 3, r=-0.072$ and $\mathrm{P}=0.652)$. Similar results were observed for bilateral TKA (on POD 1, $r=0.238$ and
$\mathrm{P}=0.286$; on POD 3, $r=0.374$ and $\mathrm{P}=0.087$ ). For U-UKA, the results of the correlation analyses demonstrated that the $\Delta \mathrm{Hb}$ levels on POD 1 were not associated with the $\Delta \mathrm{SpO}_{2}$ levels $(r=-0.164 ; \mathrm{P}=0.792)$. However, the opposite was observed for POD 3 ( $r=0.942 ; \mathrm{P}=0.017)$, which may have been due to the relatively small sample size of the U-UKA group. 


\section{Discussion}

Due to the lack of consistency in the literature with regard to the definition and monitoring of hypoxemia, marked discrepancies in reported incidences of hypoxemia following arthroplasty have been found. The incidence of hypoxia in clinical settings is generally unknown. A previous study reported a $42 \%$ incidence of hypoxemia on POD 1 following THA (7). Austin et al (3) demonstrated that hypoxemia occurred more frequently during POD 2, and that its incidence was relatively low (4\%). Hong et al (13) reported a case of hypoxemia in a healthy elderly male patient that developed $2 \mathrm{~h}$ following cementation of the prosthesis during THA under spinal anesthesia. However, increases in ventilation-perfusion mismatch were demonstrated by Ereth et al (14) to occur within $30 \mathrm{~min}$ following insertion of the femoral prosthesis. The lowest $\mathrm{SpO}_{2}$ value was shown to often occur on the day of surgery, with significant hypoxemia on POD 0-2 followed by a stable increase in the first 3-5 days (14). However, Mimura et al (15) reported that even on POD 5, oxygen tension was decreased by $>10 \%$ in 5 of the 10 post-TKA patients. It was also noted that none of these patients developed clinical dyspnea, and only one patient was diagnosed with pulmonary embolism using a ventilation/perfusion scintigraphy (15).

Postoperative hypoxemia results most frequently from one of two possible physiological mechanisms: Hypoventilation or abnormal gas exchange (16). These mechanisms include pulmonary embolism, pulmonary edema, and pneumonia. Hypoxemia also varies according to the physical status and cardiovascular reserve of the patient $(7,16)$. Postoperative hypoxemia can be secondary to numerous conditions $(1,3,7)$, including the effects of narcotics, postoperative atelectasis, fluid overload, and hypoventilation. Blood loss following revision surgery requires abundant blood transfusion and high fluid resuscitation (9). Intravenous fluid overload in a weak, malfunctioning heart predisposes a patient to pulmonary edema, which may result in respiratory failure and hypoxemia (3). In the present study, a significant difference was identified in $\Delta \mathrm{SpO}_{2}$ between hip and knee arthroplasties. The results of previous studies suggested that a thrombogenic process is created from venous stasis and intimal wall damage caused by tourniquet use $(17,18)$. Hypoxemia may result from venous embolization that occurs in conjunction with intramedullary hypertension in the femur during insertion of the prosthesis in patients undergoing arthroplasty (14). A second explanation is based on the results of transesophageal echocardiographic studies that demonstrated that fat embolic loads are greater following cemented TKA than following uncemented THA (19). Within knee arthroplasty, the incidence of filling defects was greater when both the femoral and tibial canals were cannulated than when only one or no bone was cannulated $(17,20)$. These results are concordant with those of a previous study which examined lower cardiac embolic load with computer-assisted surgery (20). Perioperative monitoring for embolism may be helpful in assessing patients in whom cardiorespiratory function deteriorates. Due to the lack of a validated screening tool $(17,21)$, the poor sensitivity of the clinical signs and symptoms of thromboembolic complications, and the possibility of a fatal outcome, clinical decision-making on the course of action for the hypoxemic patient is difficult (3). The value of pulse oximetry and blood gases in evaluating pulmonary embolism remains unclear. Episodic hypoxemic events have been described in sleeping postoperative patients who had received analgesic therapy such as opioids (22). In addition, patients with obstructive sleep apnea had a higher and more transient hypoxia; however, the incidence of complications experienced by these patients was not increased following appropriate oxygen supplementation $(4,23)$.

The results of the present study indicated that $\Delta \mathrm{SpO}_{2}$ was inconsistent with a decrease in $\mathrm{Hb}$ levels. There appeared to be little correlation between the two random variables. Arthroplasty is usually associated with significant blood loss $(9,10)$. Documented complications of perioperative anemia include tachycardia, hypotension, and increased risk of perioperative myocardial infarction (24). The oxygen-carrying capacity of blood is likely best determined by the mass of circulating red blood cells $(3,16,25)$. Anemia is a condition characterized by a decrease in the oxygen-carrying capacity of blood. Under most circumstances, Hb concentration is a good indicator of red cell mass, but changes in plasma volume may lead to discrepancies in the results. In patients undergoing surgical procedures and critically ill patients, fluctuations in the plasma volume often occur due to the use fluid resuscitation to treat patients with fluid resuscitation for hypovolemia and increased capillary leakage (25). Some investigators have hypothesized that anemia-induced tissue hypoxia elicits the same responses as exposure to low fractions of inspired oxygen or systemic hypoxia $(26,27)$. There are important differences between anemia and hypoxia in terms of blood chemistry (28). During anemia, a reduction in blood oxygen content occurs as a result of reduced $\mathrm{Hb}$ levels while arterial oxygenation and oxyhemoglobin saturation remain high. During hypoxia, arterial oxygenation is reduced, which results in oxyhemoglobin desaturation at normal $\mathrm{Hb}$ levels (26). At reduced $\mathrm{Hb}$ levels, optimal oxygen loading of $\mathrm{Hb}$ in the lung is observed with anemia (29). The mechanisms underlying these effects are thought to include a nitric oxide-mediated increase in minute ventilation and ventilation/perfusion matching, which optimizes $\mathrm{Hb}$ saturation to $\sim 100 \%$ (29). $\mathrm{SpO}_{2}$ serves as a cardiopulmonary oxygen transfer measurement, not a measure of tissue perfusion. A severely anemic person may have an $\mathrm{SpO}_{2}$ of $100 \%$ but a very low oxygen-carrying capacity. This is the cause of anemia-associated problems with wound healing, organ failure and other complications $(9,24)$. Severe anemia is associated with marked stability of pulmonary gas exchange (30). Anemia results in improved gas exchange in the normal lung due to an improvement in overall ventilation/perfusion matching. In turn, this may be a result of favorable changes in pulmonary blood flow distribution, as assessed by the fractal dimension and spatial correlation of blood flow and as a result of increased nitric oxide availability $(26,27)$. The increase in cardiac output results from a composite increase in both heart rate and stroke volume, which acts to increase cardiac output and thereby maintain overall systemic oxygen delivery and consumption with low Hb levels $(31,32)$.

There were limitations to the present study. The majority of the patients in the study underwent general anesthesia, which may account for the perioperative decrease in $\mathrm{SpO}_{2}$ levels. Medical comorbidities were not considered in the analysis. The sample sizes were relatively small, since there were numerous type of surgical procedures and deep venous 
thrombosis prophylaxis and anesthesia that were used. Further studies with larger cohorts are required in order to draw conclusions regarding blood loss.

In conclusion, $\mathrm{SpO}_{2}$ levels should not serve as a clinical indicator of the incidence and severity of anemia in patients who have undergone primary arthroplasty. To a point, postoperative anemia does not affect $\mathrm{SpO}_{2}$ levels. Hypoxemia alone was not found to be specific for a particular etiology, and further diagnostic studies are required.

\section{Acknowledgements}

The authors of the present study would like to thank the Department of Epidemiology and Bio-statistics at the School of Public Health, Peking University (Beijing, China) for assistance with statistical analyses, and to Miss Fan Meng at the China-Japan Friendship Hospital for help with data collection. The present study was supported by grants from the National Natural Science Foundation of China (grant no. 81372013) and the Research Fund of the China-Japan Friendship Hospital (grant nos. 2013-MS-27 and 2014-4-QN-29).

\section{References}

1. Galway UA and Gugliotti D: Sudden hypoxia during knee surgery. Cleve Clin J Med 79: 401-409, 2012.

2. Clarke MT, Longstaff L, Edwards D and Rushton N: Tourniquet-induced wound hypoxia after total knee replacement. J Bone Joint Surg Br 83: 40-44, 2001.

3. Austin L, Pulido L, Ropiak R, Porat M, Parvizi J and Rothman RH: Hypoxemia after total joint arthroplasty: A problem on the rise. J Arthroplasty 23: 1016-1021, 2008.

4. Lewer BM, Larsen PD, Torrance JM and Galletly DC: Artefactual episodic hypoxaemia during postoperative respiratory monitoring. Can J Anaesth 45: 182-185, 1998.

5. Gill NP, Wright B and Reilly CS: Relationship between hypoxaemic and cardiac ischaemic events in the perioperative period. Br J Anaesth 68: 471-473, 1992.

6. Lin GM, Chen YJ, Li YH, Jaiteh LE and Han CL: The effect of hypoxia-hypercapnia on neuropsychological function in adult respiratory distress syndrome. Am J Respir Crit Care Med 186: 1307-1308, 2012.

7. Clayer M and Bruckner J: Occult hypoxia after femoral neck fracture and elective hip surgery. Clin Orthop Relat Res 265-271, 2000.

8. Freeman TA, Parvizi J, Dela Valle CJ and Steinbeck MJ: Mast cells and hypoxia drive tissue metaplasia and heterotopic ossification in idiopathic arthrofibrosis after total knee arthroplasty. Fibrogenesis Tissue Repair 3: 17, 2010.

9. Sehat KR, Evans RL and Newman JH: Hidden blood loss following hip and knee arthroplasty. Correct management of blood loss should take hidden loss into account. J Bone Joint Surg Br 86: 561-565, 2004.

10. Parvizi J, Pour AE, Peak EL, Sharkey PF, Hozack WJ and Rothman RH: One-stage bilateral total hip arthroplasty compared with unilateral total hip arthroplasty: A prospective study. J Arthroplasty 21 (6 Suppl 2): S26-S31, 2006.

11. Shephard DA: The 1975 Declaration of Helsinki and consent. Can Med Assoc J 115: 1191-1192, 1976.

12. Gao F, Guo W, Sun W, Li Z, Wang W, Wang B, Cheng L: Mechanisms and influencing factors of hypoxemia after joint arthroplasty. Zhonghua Yi Xue Za Zhi 94: 655-659, 2014 (In Chinese).

13. Hong CL, Liu HP, Wu CY, Ho AC, Shyr MH, Wong CH and Chun HS: Delayed hypoxemia after bone cement insertion during total hip replacement under spinal anesthesia-a case report. Acta Anaesthesiol Sin 41: 47-51, 2003.
14. Ereth MH, Weber JG, Abel MD, Lennon RL, Lewallen DG, Ilstrup DM and Rehder K: Cemented versus noncemented total hip arthroplasty-embolism, hemodynamics, and intrapulmonary shunting. Mayo Clin Proc 67: 1066-1074, 1992.

15. Mimura $M$, Yamazaki Y, Yamamoto $H$ and Namiki A: Postoperative hypoxia and hyperfibrinolysis in patients after total knee replacement. Masui 47: 190-194, 1998 (In Japanese).

16. Jones JG, Sapsford DJ and Wheatley RG: Postoperative hypoxaemia: Mechanisms and time course. Anaesthesia 45: 566-573, 1990.

17. Gandhi R, Salonen D, Geerts WH, Khanna M, McSweeney S and Mahomed NN: A pilot study of computed tomography-detected asymptomatic pulmonary filling defects after hip and knee arthroplasties. J Arthroplasty 27: 730-735, 2012.

18. Kato N, Nakanishi K, Yoshino S and Ogawa R: Abnormal echogenic findings detected by transesophageal echocardiography and cardiorespiratory impairment during total knee arthroplasty with tourniquet. Anesthesiology 97: 1123-1128, 2002.

19. Hagio K, Sugano N, Takashina M, Nishii T, Yoshikawa H and Ochi T: Embolic events during total hip arthroplasty: An echocardiographic study. J Arthroplasty 18: 186-192, 2003.

20. Church JS, Scadden JE, Gupta RR, Cokis C, Williams KA and Janes GC: Embolic phenomena during computer-assisted and conventional total knee replacement. J Bone Joint Surg Br 89: 481-485, 2007.

21. Lawton RL, Morrey BF and Narr BJ: Validity of index of suspicion for pulmonary embolism after hip arthroplasty. Clin Orthop Relat Res 180-192, 2003.

22. Catley DM, Thornton C, Jordan C, Lehane JR, Royston D and Jones JG: Pronounced, episodic oxygen desaturation in the postoperative period: Its association with ventilatory pattern and analgesic regimen. Anesthesiology 63: 20-28, 1985.

23. Berend KR, Ajluni AF, Núñez-García LA, Lombardi AV and Adams JB: Prevalence and management of obstructive sleep apnea in patients undergoing total joint arthroplasty. J Arthroplasty 25 (Suppl 6): S54-S57, 2010

24. Liu X, Zhang X, Chen Y, Wang Q, Jiang Y and Zeng B: Hidden blood loss after total hip arthroplasty. J Arthroplasty 26: 1100-1105.e1, 2011.

25. McLellan SA, McClelland DB and Walsh TS: Anaemia and red blood cell transfusion in the critically ill patient. Blood Rev 17: 195-208, 2003.

26. Hare GM, Tsui AK, Ozawa S and Shander A: Anaemia: Can we define haemoglobin thresholds for impaired oxygen homeostasis and suggest new strategies for treatment? Best Pract Res Clin Anaesthesiol 27: 85-98, 2013.

27. Hare GM, Freedman J and David Mazer C: Review article: Risks of anemia and related management strategies: Can perioperative blood management improve patient safety? Can J Anaesth 60: 168-175, 2013.

28. Tsui AK, Marsden PA, Mazer CD, Adamson SL, Henkelman RM, Ho JJ, Wilson DF, Heximer SP, Connelly KA, Bolz SS, et al: Priming of hypoxia-inducible factor by neuronal nitric oxide synthase is essential for adaptive responses to severe anemia. Proc Natl Acad Sci USA 108: 17544-17549, 2011.

29. Deem S, Hedges RG, McKinney S, Polissar NL, Alberts MK and Swenson ER: Mechanisms of improvement in pulmonary gas exchange during isovolemic hemodilution. J Appl Physiol (1985) 87: 132-141, 1999.

30. Deem S, Alberts MK, Bishop MJ, Bidani A and Swenson ER: $\mathrm{CO} 2$ transport in normovolemic anemia: Complete compensation and stability of blood CO2 tensions. J Appl Physiol (1985) 83: 240-246, 1997

31. Weiskopf RB, Viele MK, Feiner J, Kelley S, Lieberman J, Noorani M, Leung JM, Fisher DM, Murray WR, Toy P and Moore MA: Human cardiovascular and metabolic response to acute, severe isovolemic anemia. JAMA 279: 217-221, 1998.

32. Ragoonanan TE, Beattie WS, Mazer CD, Tsui AK, Leong-Poi H, Wilson DF, Tait G, Yu J, Liu E, Noronha M, et al: Metoprolol reduces cerebral tissue oxygen tension after acute hemodilution in rats. Anesthesiology 111: 988-1000, 2009. 